\title{
Oxytocin Receptor-Expressing Neurons and Nuclei in the Regulation of Social Behaviors
}

\author{
Katsuhiko NISHIMORI ${ }^{1, *}$, Keisuke SATO $^{1}$, Shizu HIDEMA ${ }^{1}$, \\ Masahide YOSHIDA ${ }^{1, \dagger}$, and Hiroaki MIZUKAMI ${ }^{2}$ \\ ${ }^{1}$ Laboratory of Molecular Biology, Department of Molecular and Cell Biology, \\ Graduate School of Agricultural Science, Tohoku University, Sendai 981-8555, Japan \\ ${ }^{2}$ Center for Molecular Medicine, Division of Genetic Therapeutics, \\ Jichi Medical University, Shimotsuke, Tochigi 329-0498, Japan
}

Received August 14, 2015; final version accepted August 16, 2015

\begin{abstract}
We previously generated oxytocin (OXT)-deficient mice and oxytocin receptor (OXTR)-deficient mice. Impaired social behaviors were observed in these mice, so they may be useful as animal models for studying the regulatory mechanism of social behavior by the OXT/OXTR system in the brain. In the present review, we aimed to overview our previous works to unravel the mechanism(s) by which OXTR deficiency leads to the impairment of social behaviors; for example, abnormalities in maternal behavior and/or social memory observed in mice deficient in the OXTR will be presented. By analyzing the brain of the OXTR-modified yellow fluorescent protein knock-in mice histologically, OXTR-expressing neurons were observed conspicuously in brain regions that are related to social behaviors. We focus on the characteristics of the regions containing neurons with prominent $O x t r$ gene expression in the present manuscript and discuss on the mechanisms through which OXT exerts its effects on social behaviors.
\end{abstract}

KEYWORDS: oxytocin, oxytocin receptor, GABAergic neurons, lateral septal nucleus, medial amygdaloid nucleus

\section{Introduction}

In 1994, we started a project for generating a mouse line in which oxytocin (OXT) was deficient [OXT-knockout (KO) mouse] by the gene targeting technique. The reproductive function of OXT in parturition and milk ejection was widely known, but the role of oxytocin in the regulation of social behaviors had not been studied in mice by that time. During the last two decades, functional roles of OXT and oxytocin receptors (OXTRs) in the brain have been explored, and the central OXT-system has been implicated in the regulation of social behaviors. Furthermore, oxytocin has been suggested to be related to the pathogenesis of certain types of psychiatric disorders including autistic spectrum disorders (ASD), and therefore, OXT and/or OXTRs are regarded potential targets for the pharmacological treatment of these disorders. Since the generation of OXT- and OXTR-deficient mice [1,2], we have carried out studies to unravel physiological roles of OXT and its receptors in the brain from an aspect of social (particularly prosocial) behavior [3-7].

\section{Identification of brain regions expressing OXT/OXTR predominantly and functional roles of these regions in animal behavior}

In the central nervous system, OXT is expressed mostly in the neurosecretory neurons in the paraventricular nucleus (PVN) and supraoptic nucleus of the hypothalamus (SON). OXT is transported through axons to the posterior lobe of the pituitary and secreted into the blood stream to control parturition and milk ejection. OXT-containing axons are also distributed extensively throughout the brain, and OXT is released from the nerve endings in multiple brain regions and exerts its effect on various neurons expressing OXTR. These brain regions are known to be related to the regulation of social behaviors and emotion, as well as other physiological functions. A reliable antibody against OXTR was unavailable for a long time, however, and detailed information as to the distribution of OXTR in the brain could not be obtained.

*Corresponding author. E-mail: knishimori@m.tohoku.ac.jp

${ }^{\dagger}$ Present address: Department of Physiology, Jichi Medical University, Shimotsuke, Tochigi 329-0498, Japan 


\section{Generation of OXTR-Venus knock-in mice and elucidation of the distribution of OXTR- expressing neurons in the brain}

OXTR-modified yellow fluorescent protein (Venus) knock-in mice were generated in our laboratory to examine the distribution of OXTR-expressing neurons in the brain and characterize their functional roles [8]. Through a detailed analysis of brains prepared from the OXTR-Venus knock-in mice, distribution of OXTR-expressing neurons was identified throughout the brain. OXTR-expressing neurons were widely distributed in mouse brain, especially in those regions which are related tightly to the regulation of social behavior and emotion [8] (Fig. 1). Based on the knowledge of the distribution of OXTR, further exploration of the OXT/OXTR system in the brain became possible.

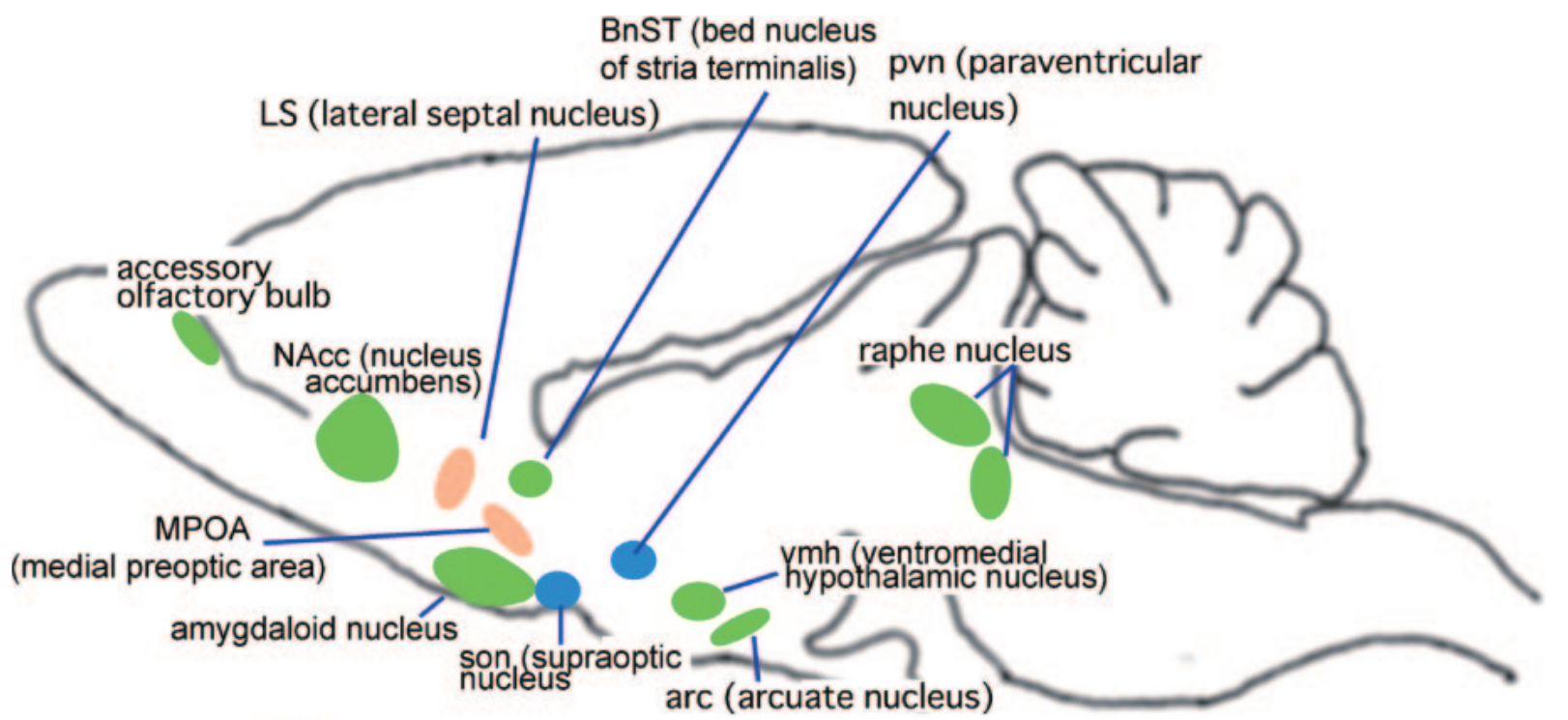

Nuclei expressing OXTR, and closely related to the regulation of social behaviors.

Nuclei expressing OXTR, and closely related to the regulation of maternal behavior.

Nuclei expressing both OXT and OXTR

Fig. 1. Localization of the regions (nuclei), expressing OXT or OXTR in mouse brain, which are related to the social behaviors and emotion Modified from Figure 1 in Katsuhiko Nishimori, Hikaku Naibunpitsugaku Vol. 39, 2013, pp. 90-95.

\section{OXTR-expressing neurons in brain regions that are related to the control of social behavior}

OXTR is expressed in different neuronal types [8] depending on the regions where it is expressed; for example, it is expressed conspicuously in serotonergic (5-HT) neurons in the raphe nucleus, gamma-aminobutyric acid (GABA)containing (GABAergic) neurons in the medial amygdaloid nucleus (MeA) and lateral septum (LS), dopaminergic neurons in the nucleus accumbens, and glutamatergic neurons in the hippocampus (data not shown).

Since the cellular types of OXTR-expressing neurons were so diverse, the mechanism through which OXT takes part in mediating or modulating the neuronal circuits may differ significantly between brain regions. However, accumulated data obtained by our group suggest strongly that the major functional effector of neurons expressing OXTR, which controls social memory and social buffering, are the GABAergic neurons in the LS and the MeA $[9,10]$.

The medial preoptic area (MPOA) is a well-known nucleus related to the control of maternal behavior. The involvement of the LS and the bed nucleus of the stria terminalis (BnST) has also been reported in the regulation of maternal behavior [11-13]. In these nuclei, activation of c-Fos and expression of OXTR were analyzed in the brains of the OXTR-Venus knock-in mice (Figs. 2, 3).

The OXTR-expressing neurons were also observed in other brain regions involved in homeostatic maintenance and behavioral regulations; these regions include the medial amygdaloid nucleus (MeA) (handling social memory), nucleus accumbens (regulation of pair bonding in prairie voles) [14], arcuate nucleus (ARC) (food intake), and hypothalamic areas (control centers for stress, body temperature, and body fluid and electrolytes) [8] (Fig. 1). We focused on clarifying the function of OXTR in the context of maternal behavior and social memory, among other social and prosocial behaviors, because the KO mice, which were generated in our laboratory (OXT-KO- and OXTR-KO mouse), 
A

VVenus(OXTR) Vc-Fos Vco-localization
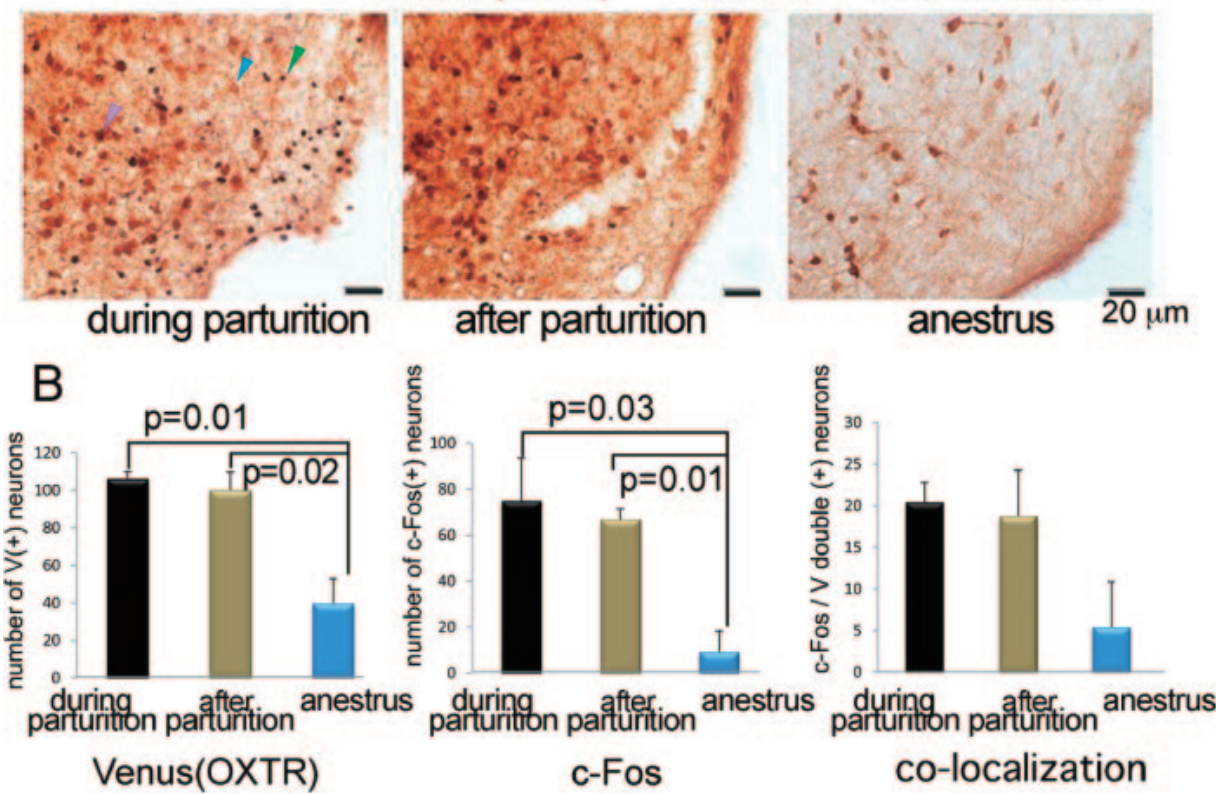

Fig. 2. The MPOA neurons expressing OXTR: changes in the number of OXTR-expressing neurons in response to neural activation during parturition and at the period when maternal behavior was initiated A, Representative slices containing the MPOA of an OXTR-Venus knock-in (Venus/+) mice, during parturition, after parturition, and at anestrus stage (as controls). The slices were immunostained with anti-c-Fos-, or anti-EGFP (Venus) antibody. B, Numbers of neurons expressing either OXTR (Venus), c-Fos, or OXTR (Venus)/c-Fos (double positive) are shown. Reproduced from Figure 2 in Katsuhiko Nishimori, Hikaku Naibunpitsugaku Vol. 39, 2013, pp. 90-95.

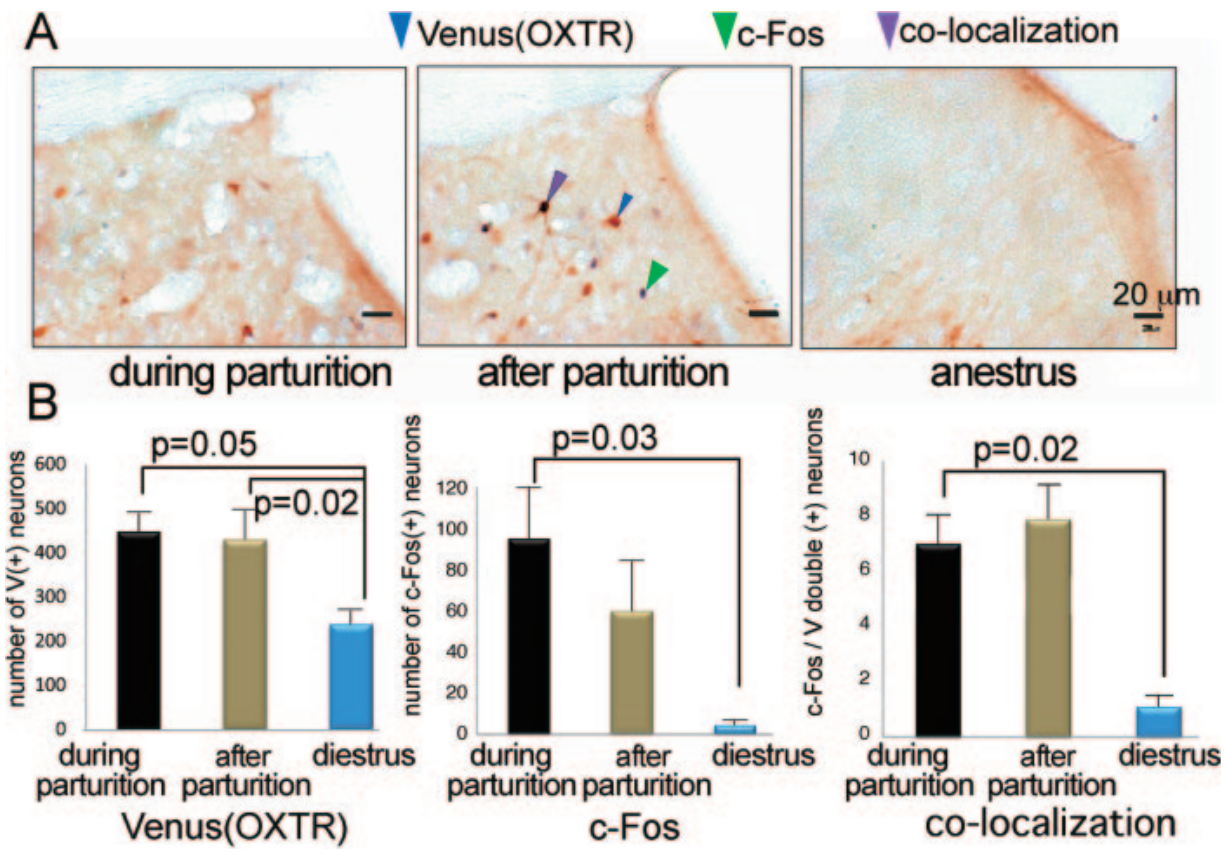

Fig. 3. The LS neurons expressing OXTR: changes in the number of OXTR-expressing neurons in response to neural activation during parturition and at the period when maternal behavior was initiated $\mathrm{A}$, Representative slices containing the LS of an OXTR-Venus knock-in (Venus/+) mice, during parturition, after parturition, and at anestrus stage (as controls). The slices were immunostained with anti-c-Fos-, or anti-EGFP (Venus) antibody. B, Numbers of neurons expressing either OXTR (Venus), c-Fos, or OXTR (Venus)/c-Fos (double positive) are shown. Reproduced from Figure 3 in Katsuhiko Nishimori, Hikaku Naibunpitsugaku Vol. 39, 2013, pp. 90-95.

showed impairment in maternal behavior and social memory $[2,9,10,15]$. Thus, the roles of OXTR-expressing neurons in the regulation of social behaviors have been disclosed gradually but steadily. 


\section{Functional brain regions related to the social behavior}

\subsection{Maternal behavior and OXTR}

Impairments in maternal behavior were observed in the OXTR-KO mice [2]. First, changes in Oxtr gene expression were monitored in brain regions, which were reported to be tightly related to the regulation of maternal behavior and social memory, by prompting these behaviors. The MPOA, LS, BnST, SON and PVN, in which predominant OXTRexpression was observed, were also related to the regulation of the maternal behavior (Fig. 1). In the brains of pregnant mice, increased expression of c-Fos was observed in the MPOA and LS regions, where we also detected increases in OXTR expression during and just after parturition, when maternal behavior was initiated. The relative ratio of the OXTR-expressing neurons that co-express c-Fos also increased in these nuclei during and after parturition (Figs. 2, 3).

\subsection{Social memory and OXTR}

Both OXT-KO mice and the OXTR-KO mice presented impairment in social memory $[2,15]$ in habituationdishabituation and social discrimination paradigms. We analyzed expression levels of c-Fos and OXTR in the brain regions postulated to mediate social memory when mice were exposed to social interactions. In these interactions, individually housed male mice were exposed to the ovariectomized female mice. Ninety minutes after the social interactions, when the activity of c-Fos was at its maximum, brains were perfused and cut, and the brain slices were immunostained with specific antibodies. The activated regions by social interactions coincided with those areas which are related to the control of social behaviors. More specifically, activation of c-Fos expression was observed in the MeA of socially exposed OXTR-Venus knock-in mice (heterogeneous OXTR knock-in mice), and a considerable proportion of the c-Fos-expressing neurons co-expressed OXTR. In contrast, in the OXTR-Venus knock-in mice (homogeneous knock-in mice, namely, OXTR-KO mice), the results obtained with heterogeneous OXTR knock-in mice could not be reproduced (data not shown).

\section{Generation of the region-specific OXTR-KO mice}

\subsection{Generation of the region-specific OXTR-KO mouse and subsequent rescue of Oxtr gene}

A novel line of OXTR-KO mice was generated in which Oxtr gene was knocked out in a region specific manner by means of the Cre-loxP system. In addition, viral transfer of Cre recombinase, via an adeno-associated virus (AAV) vector, was employed. We also developed an AAV-OXTR-IRES-Venus vector [16] to rescue the Oxtr gene in the brain of OXTR-deficient mice in a region specific manner. The LS was analyzed for assessing maternal behaviors and the MeA was chosen for social memory. Maternal behavior, or social memory was monitored after region specific deletion of the Oxtr gene in Oxtr $(f l x / f l x)$ mice, and then region-specific rescue of the Oxtr gene in Oxtr (-/-) mice was examined.

\subsection{Behavior of female mice with LS-specific deletion of the Oxtr gene and the effect of LS-specific rescue of the Oxtr gene}

The maternal behavior of the Oxtr (-/-) mice was restored after infection with AAV-OXTR-IRES-Venus to their LS regions. In contrast, elimination of the Oxtr gene in the LS of $O x t r(f l x / f l x)$ mice, by infection of AAV-Cre, did not show any impairment in their maternal behaviors (unpublished observations).

\subsection{Social memory of male mice with MeA specific elimination of OXTR gene and MeA region-specific rescue of the Oxtr gene}

The social memory was impaired in Oxtr (-/-) mice, as described above, and it was restored subsequently by infection of the MeA of Oxtr (-/-) mice with AAV-OXTR-IRES-Venus vector. The social memory was also impaired by the conditional elimination of the Oxtr gene in the MeA of $O x t r(f l x / f l x)$ mice by infection with AAV-Cre to the MeA (Fig. 4) (unpublished observations). Remarkably, this impairment was similar to the behavioral phenotype with conventional Oxtr (-/-) mice. Together with the AAV-OXTR-IRES-Venus-dependent rescuing experiment (data not shown), our data suggest the significance of the Oxtr gene expressed in the MeA on social memory. We are studying further to elucidate the OXTR-mediated neural circuits, aiming at unraveling the mechanism through which the OXT/ OXTR system takes part in the regulation of social behaviors.

\section{Acknowledgments}

This work was partly supported by Grants 14360046, 20380058, and 10164609 from the Ministry of Education, Culture, Sports, Science, and Technology of Japan, and "Integrated Research on Neuropsychiatric Disorders" carried out under the Strategic Research Program for Brain Sciences by the Ministry of Education, Culture, Sports, Science, and Technology of Japan. K.S. was supported by the Japan Society for the Promotion of Science Research Fellowship 


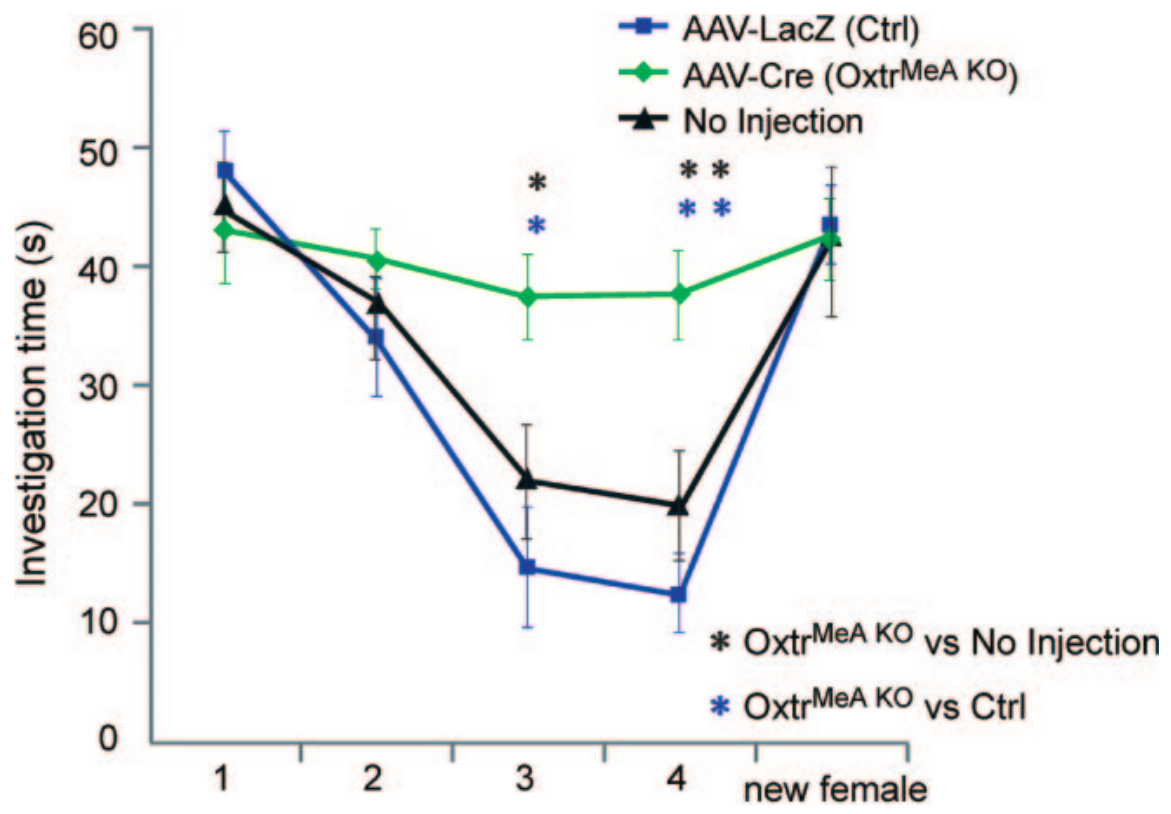

Fig. 4. Dependency of social memory formation on OXTR expression in the MeA Habituation-dishabituation test [15] was done using Oxtr $(f l x / f l x)$ male mice. AAV-Cre viral vector was injected into the MeA (MeA KO). In the control mice (Ctrl), AAV-LacZ viral vector was injected. In another group of mice, no injection of viral vectors was made (No Injection). Student's $\mathrm{t}$ test was used for statistical analysis, and a $\mathrm{P}$ value of $<0.05(*)$ was regarded as statistically significant.

for Young Scientist. The Authors thank Daichi Osada, Yutaro Chiba and Emi Asayama for their partial contribution in this work. Disclosure Summary: The authors have nothing to disclose.

\section{REFERENCES}

[1] Nishimori K, Young LJ, Guo Q, Wang Z, Insel TR, Matzuk MM (1996) Oxytocin is required for nursing but is not essential for parturition or reproductive behavior. Proc Natl Acad Sci USA 93:11699-11704.

[2] Takayanagi Y, Yoshida M, Bielsky IF, Ross HE, Kawamata M, Onaka T, Yanagisawa T, Kimura T, Matzuk MM, Young LJ, Nishimori K (2005) Pervasive social deficits, but normal parturition, in oxytocin receptor-deficient mice. Proc Natl Acad Sci USA 102:16096-16101.

[3] Kasahara Y, Takayanagi Y, Kawada T, Itoi K, Nishimori K (2007) Impaired thermoregulatory ability of oxytocin-deficient mice during cold-exposure. Bioscience, Biotechnology, and Biochemistry 71:3122-3126.

[4] Takayanagi Y, Kasahara Y, Onaka T, Takahashi N, Kawada T, Nishimori K (2008) Oxytocin receptor-deficient mice developed late-onset obesity. Neuroreport 19:951-955.

[5] Kasahara Y, Sato K, Takayanagi Y, Mizukami H, Ozawa K, Hidema S, So K-H, Kawada T, Inoue N, Ikeda I, Roh S-G, Itoi K, Nishimori K (2013) Oxytocin receptor in the hypothalamus is sufficient to rescue normal thermoregulatory function in male oxytocin receptor knockout mice. Endocrinology 154:4305-4315.

[6] Tamma R, Colaianni G, Zhu L, DiBenedetto A, Greco G, Montemurro G, Patano N, Strippoli M, Vergari R, Mancini L, Colucci S, Grano M, Faccio R, Liu X, Li J, Usmani S, Bachar M, Bab I, Nishimori K, Young L, Buettner C, Iqbal J, Sun L, Zaidi M, Zallone A (2009) Oxytocin is an anabolic bone hormone. Proc Natl Acad Sci USA 106:7149-7154.

[7] Colaianni G, Sun L, Benedetto AD, Tamma R, Zhu LL, Cao J, Grano M, Yuen T, Colucci S, Cuscito C, Mancini MLJ, Nishimori K, Bab I, Lee HJ, Iqbal J, Young WS, Rosen C, Zallone A, Zaidi M (2012) Bone marrow oxytocin mediates the anabolic action of estrogen on the skeleton. J Biol Chem 287:29159-29167.

[8] Yoshida M, Takayanagi Y, Inoue K, Kimura T, Young LJ, Onaka T, Nishimori K (2009) Evidence that oxytocin exerts anxiolytic effects via oxytocin receptor expressed in serotonergic neurons in mice. J Neurosci 29:2259-2271.

[9] Guzmán YF, Tronson NC, Jovasevic V, Sato K, Guedea AL, Mizukami H, Nishimori K, Radulovic J (2013) Fear-enhancing effects of septal oxytocin receptors. Nat Neurosci 16:1185-1187.

[10] Mesic I, Guzman YF, Guedea AL, Jovasevic V, Corcoran KA, Leaderbrand K, Nishimori K, Contractor A, Radulovic J (2015) Double Dissociation of the Roles of Metabotropic Glutamate Receptor 5 and Oxytocin Receptor in Discrete Social Behaviorsm. Neuropsychopharmacology 40:2337-2346.

[11] Numan M, Stolzenberg DS (2009) Medial preoptic area interactions with dopamine neural systems in the control of the onset and maintenance of maternal behavior in rats. Front Neuroendocrinol 30:46-64.

[12] D'Anna KL, Gammie SC (2009) Activation of corticotropin-releasing factor receptor 2 in lateral septum negatively regulates maternal defense. Behav Neurosci 123:356-368.

[13] Meddle SL, Bishop VR, Gkoumassi E, van Leeuwen FW, Douglas AJ (2007) Dynamic Changes in Oxytocin Receptor Expression and Activation at Parturition in the Rat Brain. Endocrinology 148:5095-5104.

[14] Ross HE, Young LJ (2009) Oxytocin and the neural mechanisms regulating social cognition and affiliative behavior. Frontiers 
in Neuroendocrinology 30:534-547.

[15] Ferguson JN, Young LJ, Hearn EF, Matzuk MM, Insel TR, Winslowet JT (2000) Social amnesia in mice lacking the oxytocin gene. Nat Genet 25:284-288.

[16] Sato K, Date S, Aoyagi Y, Kasahara Y, Nawa A, Mizukami H, Hidema S, Ozawa K, Nishimori K (2009) Generation of adenoassociated virus vector enabling functional expression of oxytocin receptor and fluorescence marker genes using the human eIF4G internal ribosome entry site element. Biosci Biotechnol Biochem 73:2145-2148. 